\title{
Natural Current Profiles in a Tokamak
}

\author{
J. B. TAYLOR \\ Institute for Fusion Strudies \\ DOE/ET/ $53088--447$ \\ The University of Texas at Austin \\ Austin, Texas 78712 \\ DE91 000128
}

\section{DISCLAIMER}

This report was prepared as an account of work sponsored by an agency of the United States Government. Neither the United States Grovernment nor any agency thereof, nor any of their employees, makes any warranty, express or implied, or assumes any legal liability or responsibility for the accuracy, completeness, or usefulness of any information, apparatus, product, or process disclosed, or represents that its use would not infringe privately owned rights. Keference herein to any specific commercial product, process, or service by trade name, trademark, manufacturer, or otherwise does not necessarily constitute or imply its endorsement, recommendation, or favoring by the United States Government or a., agency thereof. The views and opinions of authors expressed herein do not necessarily state or reflect those of the United States Government $c i$ any agency thereof. 


\section{Introduction}

In the theory of relaxation of magnetised plasmas [1] the relaxed state, following a period of strong turbulence, is one which minimises the magnetic energy subject, only to constiant. mannetic helicity. This concept has been successful in predicting or interpreting the mannetic lickl profiles, and other features, of many plasma confinement experiments inc. including the loroidal Pinch, Spheromak and Multipinch. However, one toroidal experiment to which relaxation theory does not apply is the Tokamak - which in normal circumstances does not exhibit relaxation. In fact, relaxation in a Tokamak apparently occurs only at a so-callod disruption-when (in accord with theory) it leads to the well-known negative voltage spilie. This is presumably because only at a disruption does the Tokamak plasma experience the strong turbulence which leads to the relaxed state.

Nevertheless, despite the fact that it does not fully relax, there is some evidence that a. normal Tokamak also has a preferred "natural profile" (though the evidence is less conclusive than in the fully relaxed experiments mentioned above). There have been several attempts [2-6] to explain this natural profile in terms of some minimum principle, but these have not gained general acceptance. The reason is not that these principles lead to unreasonable profiles; rather it is because there is no obvious mechanism which would lead to the proposed state. (It is important to recognise that relaxation is not a mathematical principle involving virtual displacements, e.g., as in Hamilton's principle for the equations of motion; it refers to physical displacements which break lines of force, thereby destroying all MHD constraints except, magnetic helicity, and dissipate energy.)

In this paper $I$ show how one may arrive at a universal, or natural, family of Tokamak profiles using only accepted physical principles. These particular profiles are similar to ones proposed previously on the basis of ad hoc variational principles and the point of the present 
paper is to provide a justification for them. However in addition, the present work provides an interesting view of 'lokamak flactuations and leads to a new result a a relationship between the inward particle pinch velocity, the diffusion coefficient and the current profile.

The basic Tokanak model is described in Section 2. In Section 3 an analogy is developed between Tokamak profiles and the equilibrium of a realisable dynamical system. 'Then in Section t the equations governing the natural Tokamak profiles are derived by applying standard statistical mechanics to this analog. The profiles themselves are calculated in Section 5 and some other results of the theory are described in Section 6.

\section{Tokamak Equilibria}

For a plasma in equilibrium or moving slowly compared to Alfvén speed, the magnetic field satisfies

$$
\nabla \times(\mathbf{J} \times \mathbf{B})=0
$$

and in the conventional Tokamak ordering (large aspect ratio, low $\beta$ ) this reduces to

$$
B_{0} \frac{d J}{d z}+\mathbf{B}_{\perp} \cdot \nabla J=0
$$

where $J\left(=J_{z}\right)$ is the toroidal current. We can also write

$$
\begin{gathered}
\mathrm{B}_{\perp} \simeq \hat{\mathrm{z}} \times \nabla \psi \\
\nabla^{2} \psi=J .
\end{gathered}
$$

We take Eqs. (2)-(4) as our basic Tokamak model.

If the magnetic field is regular, with magnetic surfaces $\mathbf{B} \cdot \nabla \psi=0$, the model leads only to the standard equilibrium equation

$$
\nabla^{2} \psi=J(\psi)
$$


where $J(y)$ is any function of $\psi$. Our aim is to find which function describes the natural protiles of a Tokanak.

Suppose that, instead of possessing perfect surfaces, the magnetic field is ergodic in the torus. Then strictly (since Eq. (2) implies that $J$ is constant along B) the (current must, be uniform throughout the torus. This is indeed the fully relaxed state of a Tokamak (in the large aspect-ratio limit) and accords with the concept that turbulence leads to breaking of all magnetic surfaces. However if the "ergodisation" of the field is small (so that a field line" moves only slowly away from the ideal surface) then uniform- $J$ is an unrealistic interpretation of E(1. (2). In order that Eq. (2) lead to a uniform current it is necessary that the field line (there is only one in an ergodic field!) should effectively explore the whole torns; if this talies many circuits of the torus then small effects omitted from Eq. (2) cause the field line to "lose its memory" of $J$ before it has explored the discharge much beyond the neighborhoor of its starting surface. Thus we should interpret Eq. (2) not in a torus---in which the field line explores a fixed volume---but in an infinite cylinder in which the field line continuously explores new local regions. Then if the mean current is non-uniform, a chaotic field $\mathbf{B}$ will lead to filamentary currents (by mixing different values of $J$ ). As one follows one of these filaments along $B$ its area and density remain constant but its cross-section becones more: and more convoluted-until small effects omitted from Eq. (2) cause it to diffuse or decay. At the same time fresh filaments are being generated by the action of the chaotic $\mathrm{B}$ on the mean current gradient. This, of course, is precisely the "Clump" picture of M.H.D. turbulence described and analysed in detail by Tetreault [7]. Nevertheless, as we will see, the present results differ from those of the Clump theory.

\section{The Dynamical Model}

In what follows we need to extract from the above picture only the concept that the fieid is slightly chaotic, without exact magnetic surfaces, and that correspondingly the current is 
filamented. The magnitude and number of filaments (which we assume to be microscopic) is not required. For simplicity we assume that they all have similar magnitude jo. There is then one last conceptual step; if we regard the (infinite) variable $\approx$ as a psendo-time $t$, and regard $\mathrm{B}_{\perp}$ as a velocity, then the equations for the $z$-dependence of the filamsented current, in a Tokamak are identical with those which describe the time evolution of a two-dimensional assembly of parallel straight rods interacting according to the (Hamiltonian) equations

$$
\begin{gathered}
j_{0} \frac{d x_{i}}{d t}=\frac{1}{B_{0}} \frac{\partial H}{\partial y_{i}} \quad j_{0} \frac{d y_{i}}{d t}=-\frac{1}{B_{0}} \frac{\partial H}{\partial x_{i}} \\
H=\sum_{i<k} j_{0}^{2} l \quad\left(\mathbf{r}_{i}, \mathbf{r}_{k}\right),
\end{gathered}
$$

where $\mathbf{r}=(x, y)$ denotes the position of the rods and $U\left(\mathbf{r}_{i}, \mathbf{r}_{k}\right)$ is the potential of a unit strength rod at $\mathbf{r}_{i}$ due to another unit rod at $\mathbf{r}_{k}$. (In an infinite region $U=\log \left|\mathbf{r}_{i}-\mathbf{r}_{k}\right|$ but we specifically need to consider a bounded system so we leave (I unspecified at present.) Note that apart from a scale factor (the length of a rod) the Hamiltonian is the energy of the system and energy is conserved. Also in terms of Eqs. (2)-(4), the flux $\psi(r)=$ $\sum_{k} j_{0} l^{i}\left(\mathbf{r}, \mathbf{r}_{k}\right)$.

Equations $(6,7)$ have another, more easily visualised, interpretation. Apart from a change of sign in the Hamiltonian, they are identical with the equations of motion for a set of parallel electrically charged rods moving with $E \times B$ velocities in a strong parallel magnetic field [8,9]. (We could even restore the sign if we envisaged that the rods also interacted gravitationally and that the gravitational interaction exceeded the electrostatic interaction!)

The important point in this discussion is that the Tokamak equilibrium problem has an exact analog in the evolution of a mechanical system. We can therefore apply the methods of conventional statistical mechanics. (However it is important not to lose sight of the fact that Eqs. $(6, \%)$ do not describe the time evolution of the Tokamak. They describe the $z$-dependence of the Tokamak current filaments at a fixed time. 


\section{The Statistical Mechanics}

We can apply the principles of statistical mechanics to our model in several ways. We may adopt the microcanonical ensemble (appropriate since the energy is conserved) when the joint probability distribution of the filaments is given by

$$
\rho\left\{\mathbf{r}_{i}\right\} \sim \delta\left[E-H\left\{\mathbf{r}_{i}\right\}\right]
$$

where $\left\{r_{i}\right\}=\left(r_{1}, r_{2}, \ldots r_{N}\right)$. Then the average density of filaments is

$$
\langle\rho(\mathbf{r})\rangle \sim \int \delta\left[E-H\left(\mathbf{r}, \mathbf{r}_{2}, \mathbf{r}_{3}, \ldots \mathbf{r}_{n}\right)\right] \prod_{2}^{N} d \mathbf{r}_{i}
$$

As usual we can divide phase space up into small cells $\Delta_{i}$, represent a given configuration $\left\{r_{i}\right\}$ by the number $n_{i}$ of filaments in each cell, and treat $n_{i}$ as large. Then

$$
\langle\rho(\mathbf{r})\rangle \sim \sum_{\text {all } n_{1}} e^{-\Sigma_{1} n_{1} \log n_{1}} \delta\left[E-\Sigma j_{0}^{2} n_{i} U_{i, j} n_{, j}\right] \delta\left[N-\Sigma n_{i}\right]
$$

Again making the conventional assumption that when $N$ is large the major contribution comes from the largest terms, we get for the mean current distribution

$$
J(\mathbf{r})=j_{0}\langle\rho(\mathbf{r})\rangle=K \exp \left(-\mu j_{0} \psi(\mathbf{r})\right)
$$

where

$$
\psi(\mathbf{r})=\int j_{0} U\left(\mathbf{r}, \mathbf{r}^{\prime}\right) \rho\left(\mathbf{r}^{\prime}\right) d \mathbf{r}^{\prime}
$$

is the potential, or flux, and $\mu$ arises as a Lagrange multiplier.

The calculation above was actually described many years ago by Montgomery, Turner and Vahala[10] in a pioneering paper. They considered filamentis of $J$ and $B$ (but as a representation of smooth profiles, not is physical entities) and distributed both over the cross-section. In the present analysis, motivated by the dynamical analogs, only the current. filaments are distributed. 
An alternative application of statistical mechanies would be to involie a "meatl-ficld" approximation in which each filanent or rod moves in the mean ficted of all the others. In this case the canonical distribution is appropriate and one obtains immediately the mean distribution

$$
J(\mathbf{r})=K \exp \left(-\beta j_{0} \psi(\mathbf{r})\right)
$$

where 3 is now the inverse "temperature." Note that this temperature is related to the interaction energy of the current filanents (i.e., to the magnetic field fluctuations) and lass nothing directly to do with the normal plasma temperature.

\section{Equilibrium Profiles}

From the previous sections we see that, starting from the Tokamak equilibrium Eqs. (2) -(t) and invoking only the concept that the current is, on a fine scale, filamented, we are led to natural Tokamak current profiles which are determined by

$$
J=\nabla^{2} \psi=A \exp (-\lambda \psi)
$$

Note that all specific characteristics of the current filaments are absorbed into the parameters $A$ and $\lambda$ so that the size and number of filaments is irrelevant to the profiles. For a given total current $I, \mathrm{Eq}$. (14) defines a one parameter family of profiles.

For a circular cross-section Tokamak the appropriate solution of E(p. (14) is

$$
\psi=\frac{2}{\lambda} \log \left(\frac{1+\alpha r^{2} / a^{2}}{1+\alpha}\right),
$$

where

$$
\lambda A a^{2}=\frac{8 \alpha}{(1+\alpha)^{2}}
$$

This gives a current profile

$$
J=\frac{J_{0}}{\left(1+\alpha r^{2} / a^{2}\right)^{2}}
$$

and a parabolic q-profile 


$$
4=4\left(1+\frac{c r^{2}}{a^{2}}\right)
$$

Protiles such as these have often been proposed before. In addition to the early work of Montgomery, Turner and Vahala [10], they were obtained by Biskamp [2], by Hsu and (hu1 [3], and by Kadomtsev [4] …all essentially by minimising the energy under virtual displacements $\delta \psi$ of magnetic flux with pressure and current being functions of $\psi$. Similar profiles have also been proposed by Pfirsch and Pohl [5], and by Minardi [6]. This is perhaps, therefore, an appropriate point to emphasise that our present interest lies in the underlying model for these profiles. Accordingly we examine this further in the next section and thereby derive some new results.

\section{Fluctuations and Diffusion}

We first consider the relation of the natural current profiles to the underlying filaments. 'The total current is related to the number and strength of the filaments

$$
N j_{0}=I=\pi A a^{2}(1+\alpha)
$$

and the profile is related to their effective temperature by

$$
\beta N j_{0}^{2}=\frac{8 \pi \alpha}{(1+\alpha)}
$$

Note that $\alpha \rightarrow 0$ when the effective temperature $\rightarrow \infty$. Thus, the fully relaxed state (uniform current) occurs when the effective temperature of the fluctuations is large. The current channel contracts indefinitely $(\alpha \rightarrow \infty)$ when the effective temperature approaches $N j_{0}^{2} / 8 \pi$. This range of positive effective temperatures in the Tokamak corresponds to the range of negative temperatures investigated by Edwards and Taylor [8] and by Joyce and Montgomery [9] in the corresponding electrostatic model. In the Tokamak itself, negative effective temperatures correspond to "hollow" current profiles. 
From the current protile alone we cannot determine the effective temperature or the individual filanent strength, only the combinations $N j_{0}$ and $\beta j_{0}$. However, the small sable Huctuations in the magnetic field (due to the "graniness" of the curent filaments) are given by (see Rel. s)

$$
\delta B_{k}^{2}=\left(\frac{N}{\pi a^{2}}\right)\left(\frac{j_{0}^{2}}{k^{2}}\right)
$$

so that the temperature and the filament strength could in principle be inferred from these fluctuations and the current profile.

A more interesting aspect of the filamentary model is its relation to transport in a Tokamak. If we consider a group of identified filaments ("test filaments") they will diffuse according to a Fokker-Planck Equation

$$
\frac{\partial \rho}{\partial t}=\nabla_{0}(D \nabla \rho-F \rho)
$$

Since the background filaments are in thermal equilibrium, the usual Einstein relation exists between the Fokker-Planck coefficients, that is

$$
\frac{F}{D}=\beta j_{0} \frac{\partial \psi}{\partial r}
$$

so that diffusion leads to the natural profile. In terms of this profile,

$$
\frac{F}{D}=\frac{4 \alpha r}{a^{2}}\left(\frac{1}{1+\alpha r^{2} / a^{2}}\right) \text {. }
$$

The diffusion represented by $\mathrm{Eq} .(22)$ is a macroscopic effect. However we know that on the microscopic scale the current filaments follow field lines. Now particle diffusion in a chaotic field also essentially follows field lines. Therefore the same relation must exist betwen the Fokkrir-Planck coefficients for particle diffusion as for the diffusion of test filaments. (Indeed the two sets of coefficients differ only by a particle velocity.) Thus, in the particle diffusion equation

$$
\frac{\partial n}{\partial t}=\frac{1}{r} \frac{\partial}{\partial r} r\left(D_{p} \frac{\partial n}{\partial r}-v_{p} n\right)
$$




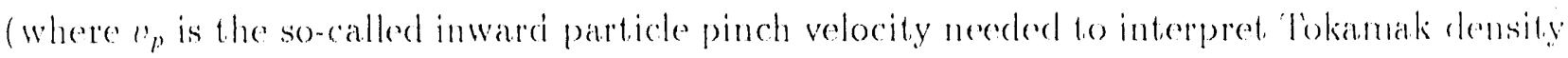
profiles) and $D_{p}$, is the particle diffusion coefficient, we have

$$
\left(\frac{a^{2} v_{p}}{r D_{p}}\right)=\frac{4 \alpha}{1+\alpha r^{2} / a^{2}}
$$

Thus, the particle diffusion is related to the current profile.

\section{Conclusions}

An exact mechanical analog exists for the Tokamak equilibrium equations if one assumes only that, because the field lines are slightly chaotic, the curcent has a microscopic filamentary structire. The time evolution of this mechanical model reflects the zadependence of the current filaments. This allows us to apply conventional statistical mechanics to find the equilibrium Tokamak current profiles.

The resulting "natural" profiles are independent of the size and number of filaments and are given in terms of a single parameter:

$$
J=\frac{J_{0}}{\left(1+\alpha r^{2} / a^{2}\right)^{2}} .
$$

Similar profiles to these have been proposed before on the basis of ad hoc variational principles.

In addition to giving a justification for these natural profiles, the present analysis provides an interesting interpretation of Tokamak fluctuations in terms of an effective temperatiure of the filaments (which has no direct connection with the true plasma temperature). Narrow profiles correspond to low effective temperature, broader, more fully relaxed profiles con'-

respond to high effective temperature, and hollow profiles correspond to negative effective temperature.

The theory described here also leads to a relation between the inward particle pinch velocity $v_{p}$ (needed to explain density profiles), the diffusion coefficient $D_{p}$, and the current. 
profile. This relation is given in Eq. (26). In terms of averaged values it, is ronghly

$$
\left\langle\frac{a v_{p}}{D_{p}}\right\rangle \sim d\left(1-\frac{q_{0}}{q_{u}}\right) .
$$

It would be interesting to see if any such relationship is observed in Tokamaks.

Finally we should note again that the picture of current filaments used here is similar to the MHD "Clump" picture of 'Tetreault [7]. However the detailed calculations of the chump. theory do not appear to lead to the profiles obtained in the present work - though they can lead to the fully relaxed state. This discrepancy requires further examination.

\section{Acknowledgement}

Part of this work was supported by the U. S. Department of Energy Contract No. DE-F(i)i8) ET T-5.3088. 


\section{References}

1. J. B. Taylor, Rev. Mod. Phys. 58, 741 (1986).

2. D. Biskamp, Comments Plasma Phys. 10, 165 (1986).

3. J. Y. Hsu and M. S. (Chu, Phys. Fluids 30, 1221 (1987).

4. B. B. Kadomtsev, Sov. J. Plasma Phys. 13, 443 (1987).

5. D. Pfirsch and F. Pohl, Plasma Phys. Contr. Fusion 29, 697 (1987).

6. E. Minardi, J. Plasma Phys. 25, 413 (1981); Plasma Phys. Contr. Fusion 31, 22!) (1989).

7. D. Tetreault, Phys. Fluids 31, 2122 (1988); Phys. Fluids B1, 511 (1989); Phys. Fluids B2, $53(1990)$.

8. S. F. Edwards and J. B. Taylor, Proc. R. Soc. Lond. A336, 257 (197.t).

9. (.. Joyce and D. Montgomery, J. Plasma Physics 10, 107 (1973).

10. D. Montgomery, L. Turner, and G. Vahala, J. Plasma Physics 21, 239 (1979). 

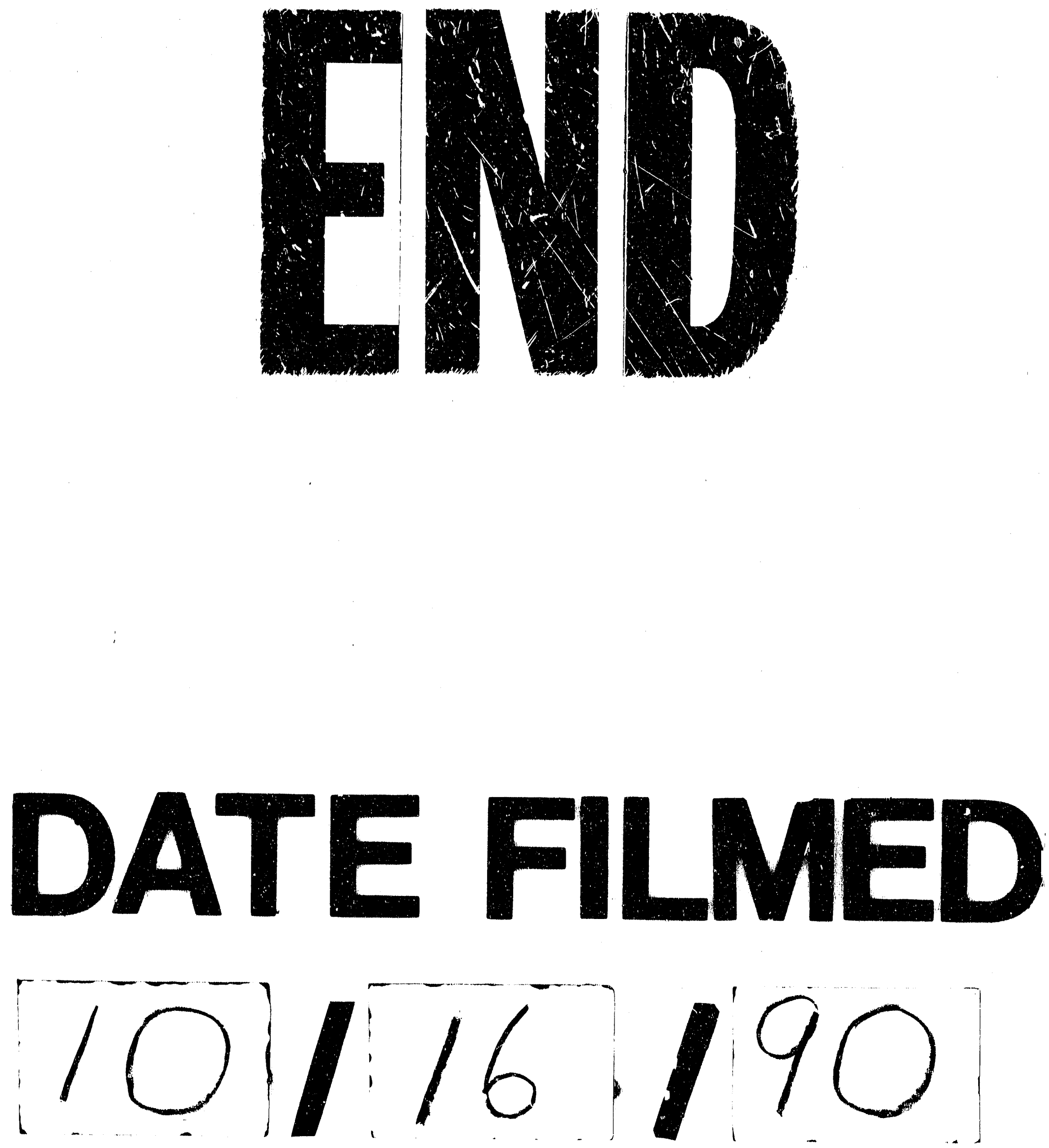
